\section{Validade de um questionário quantitativo de freqüência alimentar desenvolvido para população feminina no nordeste do Brasil}

\section{Validity of a quantitative food frequency questionnaire developed for women in the Northeast of Brazil}

Flávia Emília Leite de Lima'

Bethzabeth Slater ${ }^{2}$

Maria do Rosário D. O. Latorre

Regina Mara Fisberg ${ }^{2}$

'Curso de Nutrição, Faculdade Evangélica do Paraná

2Departamento de Nutrição, Faculdade de Saúde Pública da USP

${ }^{3}$ Departamento de Epidemiologia, Faculdade de Saúde Pública da USP

Auxílio financeiro: CNPq - Processo $\mathrm{n}^{\circ}$. 475215/01-0: Artigo baseado em: Lima FEL de. Fatores dietéticos e câncer de mama na Paraíba: um estudo caso-controle. [Tese de doutorado apresentada à Faculdade de Saúde Pública da Universidade de São Paulo]

Correspondência: Flávia Emília Leite de Lima, R Padre Anchieta 2770 (Coordenação de Nutrição), Bigorrilho, Curitiba, PR, CEP 80730-000

E-mail:flaemilia@gmail.com

\section{Resumo}

Objetivo: Verificar a validade relativa de um questionário quantitativo de freqüência alimentar (QQFA) para ser utilizado em estudo caso-controle sobre fatores dietéticos e câncer de mama no Estado da Paraíba. Métodos: A validade relativa do QQFA, com 68 itens alimentares, foi investigada em uma população de 38 mulheres, entre 25 e 80 anos, potenciais controles para o estudo principal. As participantes responderam a quatro recordatórios de 24horas (R24h), administrados por entrevistador, por telefone, em um período de um ano, e um QQFA ao final do estudo. Os valores médios de energia e nutrientes do QQFA foram comparados com a média dos quatro R24h através do teste- $t$ emparelhado. Os coeficientes de correlação de Pearson entre a média dos nutrientes antes e após o ajuste por energia nos dois métodos foram calculados e sua correção foi realizada pela variabilidade intrapessoal (coeficiente de correlação deatenuado). Resultados: A validade relativa foi verificada para energia, carboidratos, lipídios, proteínas, vitamina A e Vitamina C. Analisando os coeficientes de correlação bruta, observaram-se valores adequados de energia $(r=0,53)$ e lipídios $(r=0,40)$ e baixo para vitamina $C(r=0,08)$. Quando os coeficientes foram corrigidos pela variabilidade intraindividual, todos os nutrientes tiveram seus coeficientes consideravelmente aumentados ( $\mathrm{r}=0,36-0,67$ ). Conclusão: Os resultados sugerem que o QQFA apresentou uma validade satisfatória para ser utilizado em estudos de dieta e câncer em mulheres na Paraíba. Contudo, para seu uso em áreas geográficas ou populações distintas, procedimentos de calibração do questionário devem ser realizados anteriormente.

Palavras-chave: Validade. Questionário de frequencia alimentar. 
Abstract

Objective: To assess the relative validity of a Quantitative Food Frequency Questionnaire (QFFQ) for a case-control study on dietary factors and breast cancer in the state of Paraíba, Northeastern Brazil. Methods: The validity of a 68-item QFFQ was investigated in 38 women, ranging from 25 to 80 years old, potential controls for a case-control study. Four 24-hour dietary recalls (24HDR) were administered by an interviewer, by telephone, during the 1-year period, and a QFFQ was applied at the end of the period to compare mean nutrient intakes. Paired t-tests were applied to verify differences in the mean of nutrients in both methods. Pearson correlation coefficients on nutrient data before and after energy adjustment in both methods were calculated and within-person variability was corrected for (de-attenuated correlation coefficient). Results: Relative validity was verified for energy, carbohydrates, lipids, protein, Vitamin A and Vita$\min$ C. Satisfactory values for energy $(r=0.53)$ and lipids $(r=0.40)$ were encountered for Pearson crude correlation coefficients, but remarkably lower for Vitamin $\mathrm{C}(\mathrm{r}=0.08)$. When coefficients were corrected for within-person variability, all nutrients presented considerably higher values $(r=0.36-0.87)$. Conclusions: Results suggest that QFFQ presents a reasonably valid measure of intake for most nutrients and can be used for diet and cancer studies in women in the State of Paraíba, northeastern Brazil. Nevertheless, calibration procedures will be required for utilization in different geographical areas of Brazil .

Key Words: Validity ; Food frequency questionnaire.

\section{Introdução}

Um grande número de estudos nos últimos 30 anos tem construído uma fundamentação teórica sobre a importância da epidemiologia nutricional como campo de investigação. O foco desse campo da epidemiologia está em medir a exposição à dieta, à freqüência e à distribuição das doenças e à exposição a outros fatores que poderiam confundir essa associação ${ }^{1}$. A forma de se alimentar é influenciada por fatores como cultura, sazonalidade, condições socioeconômicas, entre outros. É na análise da dieta como fator de exposição que reside o grande desafio da epidemiologia nutricional, uma vez que esta é muito difícil de avaliar, especialmente no que concerne à ingestão usual dos indivíduos.

O Questionário de Freqüência Alimen$\operatorname{tar}(\mathrm{QFA})$ é atualmente o instrumento mais utilizado para estudos epidemiológicos que avaliam a relação entre dieta e doença, uma vez que, além de permitir avaliar a ingestão no período anterior ao aparecimento da mesma, é um método mais objetivo e menos oneroso em pesquisas de larga escala. Um dos princípios básicos da abordagem através de um QFA é a capacidade de avaliar a dieta por um longo período de tempo, considerando assim o tempo de exposição mais importante que o consumo de alimentos de alguns dias específicos².

Quando o consumo de alimentos é mensurado, faz-se necessário estimar e corrigir o erro de medição para que assim seja possível classificar de forma mais precisa os indivíduos por níveis de ingestão. Estudos de validade avaliam o grau de precisão do instrumento a ser utilizado, o que nos permite conhecer o erro e corrigir os parâmetros ${ }^{3}$.

O termo validade é geralmente definido como o grau em que um instrumento mede o que se propõe a medir ${ }^{2,4}$. Os estudos de validação consistem em comparar o resultado das estimativas do consumo de nutrientes ou de alimentos em um método de avaliação dietética escolhido pelo 
pesquisador com um outro julgado mais preciso, considerado como "padrão ouro" ou método de referência. Estudos de validação estimam parâmetros estatísticos desconhecidos dentro de um modelo que especifica relações entre a medição da ingestão e o consumo verdadeiro ${ }^{5}$.

Para a utilização de um QFA em estudos caso-controle, o ideal é que o processo de validação do questionário se faça com os potenciais controles do estudo principal $^{6}$. Os indivíduos não doentes, mas que possuem as mesmas características dos casos, são a população ideal para coletar informações sobre o consumo de alimentos a fim de verificar a validade do questionário. Essa população estaria livre dos possíveis vieses de informação que os casos poderiam apresentar.

O presente estudo examinou a validade, por telefone, de um questionário quantitativo de freqüência alimentar - QQFA desenvolvido para estudo caso-controle sobre dieta e câncer de mama no Estado da Paraíba, Brasil.

\section{Material e Métodos}

\section{População de estudo}

O presente questionário foi elaborado e validado para a investigação da relação da dieta com o câncer de mama em mulheres no Estado da Paraíba. Para a construção do instrumento, o estudo foi conduzido em uma amostra aleatória de mulheres que seriam potenciais controles para o estudo caso-controle a ser realizado ${ }^{6}$. Portanto, aquelas com idade entre 25 e 80 anos, pacientes das diversas clínicas dos ambulatórios do hospital universitário Lauro Wanderley, hospital geral onde seria realizada a coleta de dados do estudo caso-controle, foram convidadas a participar. O primeiro contato com as mulheres se deu através de convite realizado por entrevistador. Foi utilizado como critério de inclusão possuir telefone para contato. Portanto, foram incluídas na amostra mulheres que possuíssem telefone em casa ou alguma outra forma de contato telefônico (tra- balho, vizinhança, telefone público). Foram recrutadas 68 mulheres, entre as quais 30 forneceram informação incompleta, totalizando 38 participantes do estudo.

\section{Desenvolvimento do QQFA}

Para o desenvolvimento do questionário, foi obtida uma lista de alimentos coletados através de um recordatório de 24horas (R24h) aplicado a 100 mulheres entre 25 e 79 anos. O QQFA construído possui 68 itens, os quais foram divididos em grupos de alimentos,de acordo com perfil nutricional, a saber: sopas e massas, carnes e peixes, leguminosas e ovos, arroz e tubérculos, leites e derivados, molhos, vegetais, frutas, pães e biscoitos, bebidas alcoólicas, doces e sobremesas. O consumo alimentar foi referido em número de vezes por dia, semana, mês ou ano, tendo sido administrado por entrevistador. Questões sobre a ingestão de gordura visível das carnes também foram adicionadas. Para a digitação dos inquéritos alimentares foi adotado, desde o estudo piloto, o procedimento de dupla entrada dos dados, feita por digitadores distintos, a fim de eliminar os possíveis erros. Uma extensiva descrição do QQFA está relatada por Lima et al ${ }^{7}$.

\section{Coleta de dados}

A coleta de dados para esse estudo foi realizada em 12 meses, com início em novembro de 2002. A validade relativa foi avaliada através da comparação entre a medida de referência e os dados coletados pelo QQFA. Cerca de 4 a 6 semanas após o primeiro contato pessoal, foram iniciadas as entrevistas, por telefone, sendo estas não datadas previamente. Todas as entrevistas foram realizadas por nutricionistas treinadas. A repetição da coleta dos recordatórios se deu a cada 2 meses, em dias não consecutivos, abrangendo dias de semana e fim de semana, bem como perpassando todas as estações do ano, a fim de coletar dados referentes ao consumo de alimentos sazonais. 
Cada participante respondeu a cinco R24h e um QQFA. Ao final da coleta, observou-se que a maioria dos participantes não havia respondido cinco R24h. Portanto, considerou-se um mínimo de quatro R24h como o necessário para participar do estudo. A consistência dos dados e o cálculo dos nutrientes do QQFA foram realizados através do software Dietsys $4.01^{8}$. Para a análise dos R24h foi utilizado o programa Virtual Nutri 1.0 ${ }^{9}$. A base de dados utilizada para o cálculo de nutrientes em ambos os softwares foi a tabela do United States Department of Agriculture (USDA). Para a análise de alimentos regionais, utilizou-se como referências para a adição dos alimentos tabelas brasileiras de composição ${ }^{10,11}$.

\section{Análise estatística}

Inicialmente os valores médios de energia e nutrientes do QQFA foram comparados com a média dos quatro R24H através do teste- $t$ emparelhado. Todas as variáveis foram transformadas em logaritmos naturais antes da análise estatística.

O coeficiente de correlação de Pearson foi utilizado a fim de comparar os valores de energia e nutrientes do QQFA com a média dos R24h. Este coeficiente foi calculado antes e após o ajuste dos valores pela energia. Para o ajuste pela energia foi utilizado o "método residual", proposto por Willett \& Stampfer (1986) ${ }^{12}$.
Considerando também a importância da variação intra-individual na determinação da validade do instrumento, a razão entre as variâncias intra e interindividual dos R24h foi utilizada a fim de "atenuar" o coeficiente de correlação de Pearson². Todas as análises foram conduzidas utilizando-se o software estatístico SPSS $10.0^{13}$.

O projeto foi aprovado pelo Comitê de Ética e Pesquisa em Seres Humanos do Hospital Universitário Lauro Wanderley. As entrevistas por telefone foram realizadas após consentimento por escrito fornecido pelas participantes por ocasião do primeiro contato pessoal com o entrevistador antes da coleta dos dados.

\section{Resultados}

A Tabela 1 mostra as médias e desvios padrão de energia e nutrientes calculados entre o QQFA e a média dos quatro R24h. Observou-se que, para energia, o consumo estimado para o QQFA foi maior que o avaliado pelo R24h. As maiores diferenças foram percebidas comparando-se a ingestão dos micronutrientes através dos dois métodos. Avaliando-se as diferenças entre os valores médios, observa-se ter havido uma superestimativa do QQFA para todos os nutrientes estudados, com exceção da proteína.

Os coeficientes de correlação de Pearson entre os R24h e o QQFA, na forma bruta, ajustados por energia e corrigidos

Tabela 1 - Estatística descritiva e diferença de médias de energia e nutrientes calculadas entre o QQFA e a média de quatro recordatórios de 24 horas aplicados na população. João Pessoa, 2001-2002.

Table 1 - Descriptive Statistics and difference of energy and nutrient means calculated between the QQFA and the average of four 24-hour recall used in the population. João Pessoa, 2001-2002.

\begin{tabular}{lcccc}
\hline NUTRIENTES & QQFA & R24h & Diferença de médias & $\mathrm{p}$ \\
& Média $(\mathrm{dp})$ & Média $(\mathrm{dp})$ & & \\
\hline Energia $(\mathrm{kcal})$ & $1503,80(201,69)$ & $1288,81(178,00)$ & $-214,99$ & 0,00 \\
Proteínas(g) & $48,28(6,53)$ & $57,25(11,40)$ & 8,96 & 0,00 \\
Gorduras $(\mathrm{g})$ & $50,28(7,42)$ & $41,57(10,97)$ & $-8,70$ & 0,30 \\
Carboidratos(g) & $225,93(34,18)$ & $169,83(26,56)$ & 56,10 & 0,00 \\
Vitamina A (RE) & $2366,02(721,37)$ & $1369,80(770,88)$ & $-996,21$ & 0,00 \\
Vitamina C(mg) & $424,63(187,08)$ & $149,47(180,71)$ & $-275,16$ & 0,00 \\
\hline
\end{tabular}


pela variância estão apresentados na Tabela 2 . A validade relativa foi verificada para energia, carboidratos, lipídios, proteínas, vitamina A e Vitamina C. Analisandose os coeficiente de correlação bruto, observou-se valores adequados de energia $(\mathrm{r}=0,53)$ e lipídios $(\mathrm{r}=0,40)$ e baixos para vitamina C $(r=0,08)$. Quando o efeito da variabilidade intraindividual foi retirado dos coeficientes brutos, todos os nutrientes tiveram seus coeficientes consideravelmente aumentados $(r=0,36-0,67)$.

Todos os valores tenderam a decrescer após o ajuste pela energia. Foi observada uma diminuição maior que $50 \%$ em relação aos valores brutos para macronutrientes. Em contrapartida, o mesmo não ocorreu para os micronutrientes. O coeficiente de correlação, chamado "deatenuado", foi calculado para corrigir a falta de precisão nos valores médios dos recordatórios devido à variação intraindividual inerente ao método. Como pôde ser observado, essa técnica incrementou todos os valores, principalmente de carboidratos, os quais passaram de $\mathrm{r}=0,10 \mathrm{a}$ $r=0,62$ e vitamina $C(r=0,18$ a $r=0,82)$. A correção feita para os nutrientes confirma altos padrões de variância intrapessoal, característicos do nutriente examinado e também deste grupo populacional.

\section{Discussão}

Este estudo foi realizado com objetivo de validar um QQFA para posterior utili- zação em estudo do tipo caso-controle sobre fatores dietéticos e câncer de mama no Estado da Paraíba.

O QQFA avalia o consumo usual em um período específico de tempo no passado. Na prática, os estudos de validação têm mostrado que as estimativas de consumo do QQFA são comparadas com as médias de consumo realizadas por repetidas aplicações de R24h ou registros diários. Por não haver "padrão ouro" para validar um questionário de freqüência, a escolha do método dependerá do tipo de população estudada, bem como da viabilidade na aplicação.

As características do presente estudo se assemelham àquelas já desenvolvidas no país $^{15-20}$. A estrutura do questionário foi adaptada de Cardoso \& Stocco $(2000)^{21}$ e, como este, o tempo de estudo foi de 12 meses, assemelhando-se também o número de itens alimentares.

A utilização do R24h como método de referência bem como a aplicação do QQFA por entrevistador foram predominantes no país ${ }^{17-20}$, sendo também utilizados em estudos na América ${ }^{22,23}$, Europa ${ }^{24,25}$ e Ásia ${ }^{26}$.

A taxa de respostas deste estudo foi baixa, o que leva a crer que apenas as pessoas mais conscientes sobre questões de saúde participaram voluntariamente, o que poderia conduzir a um viés de autoseleção. Porém, realizou-se uma análise para avaliar o perfil das não-participantes do estudo,sendo constatado que estas pos-

Tabela 2 - Coeficientes de correlação de Pearson (ajustados e não ajustados pelo consumo de energia) entre o consumo de energia e nutrientes avaliados pelos QQFA e quatro Recordatórios de 24 horas. João Pessoa, 2001-2002.

Table 2 -Pearson correlation coefficients (adjusted and non adjusted by energy consumption) between energy and nutrient consumption assessed by the QQFA and four 24-hour Recalls. João Pessoa, 2001-2002.

\begin{tabular}{|c|c|c|c|c|}
\hline \multirow{2}{*}{$\begin{array}{l}\text { Nutrientes* } \\
\text { Energia (kcal) }\end{array}$} & \multicolumn{2}{|c|}{$\begin{array}{l}\text { Não ajustado } \\
\text { Bruto Deatenuado }\end{array}$} & \multicolumn{2}{|c|}{$\begin{array}{l}\text { Ajustado por energia } \\
\text { Bruto Deatenuado }\end{array}$} \\
\hline & $0,53^{*}$ & 0,64 & - & - \\
\hline Proteínas(g) & 0,26 & 0,57 & 0,08 & $0,17^{* *}$ \\
\hline Lipídios(g) & $0,40^{*}$ & 0,50 & $-0,16$ & $0,27^{* *}$ \\
\hline Carboidratos(g) & 0,30 & 0,67 & $-0,10$ & $0,62^{* *}$ \\
\hline Vitamina $A(R E)$ & 0,21 & 0,49 & 0,29 & $0,68^{* *}$ \\
\hline Vitamina C(mg) & 0,08 & $0,36^{* *}$ & 0,18 & $0,82^{* *}$ \\
\hline
\end{tabular}

$*<0,05$

${ }^{* *} \mathrm{p}<0.01$ 
suíam perfil semelhante as participantes. Uma baixa participação foi característica dos estudos de validação realizados no EPIC $^{24,25}$.

Uma particularidade a ser considerada foi a técnica utilizada na aplicação dos inquéritos, cujas informações foram coletadas por telefone. Essa metodologia não é recente e alguns estudos mostraram que um QQFA administrado por telefone pode dar resultados similares a entrevistas face-a-face ${ }^{27-30}$.

$\mathrm{O}$ fato de realizar entrevistas por telefone poderia introduzir um viés ao estudo, uma vez que para esse tipo de entrevista deve ser levado em conta quão bem esta amostra representa o conjunto da população que se quer avaliar. Vale salientar, portanto, que não foi condição primordial possuir telefone. Para evitar esse viés, não participaram da pesquisa apenas as pessoas com telefone em suas casas, mas também aquelas que forneceram telefone do trabalho, além do celular e telefones de vizinhos.

Outro aspecto que não pode deixar de ser considerado é que os coeficientes de correlação podem variar consideravelmente dependendo da estrutura do questionário. Subar et al. (2001) ${ }^{31}$, comparando 3 tipos de questionários de freqüência ,observaram que, após a correção pela variância todos os questionários, melhoraram suas correlações, porém aquele com estrutura semi-quantitativa, em que não variou o tamanho da porção para homens e mulheres, teve um aumento considerável, mostrando assim que, embora os três questionários apresentados sejam adequados e válidos para classificar os indivíduos de acordo com níveis de ingestão, sua estrutura pode alterar drasticamente os resultados das correlações após os devidos ajustes.

Devido à correlação existente entre energia e consumo de nutrientes, os coeficientes de correlação foram analisados antes e depois do ajuste de energia, realizado pelo método residual ${ }^{2}$. Após o ajuste, todos os coeficientes decresceram. Esse dado foi observado também em validação de questionário realizada no Brasil por Marchioni et al (2007) ${ }^{32}$. Os coeficientes de correlação tendem a aumentar quando a variabilidade na ingestão do nutriente está relacionada ao consumo de energia, mas a decrescer quando essa variabilidade depende de erros sistemáticos de super ou subestimativa $^{12}$.

Uma alteração importante foi verificada para carboidratos, vitaminas A e C após a correção pela variância, o que indica uma variabilidade intraindividual extremamente alta. O consumo desses nutrientes é significativo na população estudada, porém com uma grande flutuação dia-a-dia, o que pôde ser observado nos recordatórios aplicados em dias não consecutivos. Segundo Hunter et al. (1988) ${ }^{33}$, a variância intrapessoal em grupos de alimentos e nutrientes é refletida tanto pela variação na freqüência quanto pelo tamanho da porção consumida. Mesmo para os alimentos que possuem tamanhos de porção bem definidos (ex: pão), a quantidade consumida de um dia para outro pode levar a uma grande variabilidade no total.

O questionário tem um aceitável desempenho para classificar os indivíduos de acordo com níveis de consumo, com exceção das proteínas. Esse resultado assemelha-se ao de Slater et al. $(2003)^{20}$, cujo questionário desenvolvido para adolescentes também não mostrou bom desempenho para tal nutriente no sexo feminino. Esses pesquisadores acreditam que aspectos socioeconômicos do consumo de alimentos em populações de diferentes estratos de renda podem explicar essas baixas correlações. Esse aspecto não pôde ser controlado, uma vez que o local da coleta dos dados atende pessoas dos mais diversos estratos de renda.

Os resultados sugerem que o QQFA apresentou uma validade satisfatória para ser utilizado em estudos de dieta e câncer em mulheres na Paraíba. Contudo, para seu uso em áreas geográficas ou populações distintas, procedimentos de calibração do questionário devem ser realizados anteriormente. 


\section{Colaboradores}

F.E.L. de Lima. R.M. Fisberg e B. Slater participaram da concepção da pesquisa, elaboração dos instrumentos de coleta dos dados, revisão da literatura, análise e processamento dos dados e redação do artigo. M.R.D.O. Latorre participou da análise e do processamento dos dados.

\section{Agradecimentos}

Os autores agradecem a Profa. Dra. Maria José de Carvalho Costa, da Universidade Federal da Paraíba, pelo auxílio na operacionalização da pesquisa.

\section{Referências}

1. Sempos CT, Liu K, Ernst ND. Food and nutrient exposures: what to consider when evaluating epidemiologic evidence. Am J Clin Nutr 1999; 69(S): S1330-8.

2. Willett WC. Nutritional Epidemiology. 2nd ed. New York: Oxford University Press, 1998.

3. Slater B e Lima FEL de. Validade e reprodutibilidade dos métodos de inquérito alimentar. In: Fisberg RM, Slater B, Marchioni DML, Martini LA. Inquéritos Alimentares: Métodos e bases científicos. Atheneu: São Paulo:2005

4. Nelson M. The validation of dietary assessment. Design concepts in nutrition epidemiology. $2^{\text {nd }}$ ed. Oxford, Oxford University Express; 1997.

5. Kaaks, $\mathrm{S}$ et al. Estimating the accuracy of dietary questionnaire assessments: validation in terms of structural equation models. Stat Medicine 1994; 13: 12742.

6. BGA Commission on Nutritional Epidemiology. Recommendations for the design and analysis of nutritional epidemiologic studies with measurement errors in the exposure variables. Eur J Clin Nutr 1993; 47(Sl2): S53-7.

7. Lima FEL de, Fisberg RM, Slater B. Desenvolvimento de um questionário quantitativo de freqüência alimentar(QQFA) para um estudo caso-controle de dieta e câncer de mama em João Pessoa - PB. Rev Bras. Epidemiol 2003; 6(4):373-9.

8. Block G, Coyle LM, Hartman AM, Scoppa SM. Revision of dietary analysis software for the Health Habits and History Questionnaire. Am J Epidemiol 1994; 139: 1190-6.

9. Phillippi ST, Szarfarc SC, Latterza AR. Virtual Nutri Sistema de Análise Nutricional [programa de computador]. Versão 1.0. São Paulo; 1996.

10. Pinheiro ABV, Lacerda EMA, Benzecry EH et al. Tabela para avaliação de consumo alimentar em medidas caseiras. $4^{\mathrm{a}}$ ed. Rio de Janeiro, São Paulo, Belo Horizonte: Atheneu; 2001.
11. Philippi ST. Tabela de Composição de Alimentos: Suporte para decisão nutricional. $2^{\text {a }}$ ed. São Paulo: Coronário; 2002.

12. Willett WC, Stampfer MJ. Total energy intake: implications for epidemiological analyses. Am J Epidemiol 1986; 124: 17-27.

13. Statistical Package in Social Sciences-SPSS, version 10.0.SPSS Inc., Chicago, Illinois EUA, 1999.

14. Lopez VJ. Validez de la evaluacion de la ingesta dietetica. In: Serra Majem LI. Nutricion y Salud Publica .Métodos, bases cientificas y aplicaciones. Ed Masson: España; 1995.

15. Sichieri R, Everhart JE, Mendonça GAS. Diet and mortality from common cancers in Brazil: an ecological study. Cad Saúde Pública 1996; 12(1): 53-59.

16. Cardoso MA, Kida AA, Tomita LY, Stocco PR. Reproducibility and validity of a food frequency questionnaire among women of Japanese ancestry living in Brazil. Nutr Research 2001; 21(5): 725-33.

17. Henriques EMV. Reprodutibilidade e validade de um questionário de freqüência alimentar em mulheres de baixa renda. [Dissertação de mestrado apresentada à Universidade Estadual do Ceará]. Ceará 2001.

18. Salvo VLMA e Gimeno SGA. Reprodutibilidade e validade de um questionário de freqüência alimentar. Rev Saúde Pública 2002; 36(4): 505-12.

19. Fornés NS, Stirnghini MLF, Elias BM. Reproducibility and validity of a food frequency questionnaire for use among low-income Brazilian workers. Public Health Nutrition 2003; 6(8), 821-7.

20. Slater B, Philippi ST, Fisber RM, Latorre MRDO. Validation of a semi-quantitative adolescent food frequency questionnaire applied at a public school in São Paulo, Brazil. Eur J Clin Nutr 2003; 57: 629-35.

21. Cardoso MA, Stocco PR. Desenvolvimento de um Questionário Quantitativo de Freqüência Alimentar em imigrantes japoneses e seus descendentes no Brasil. Cad Saúde Pública 2000; 16(1): 107-114. 
22. Hernández-Avila M, Romieu I, Parra S, et al. Validity and reproducibility of a food frequency questionnaire to assess dietary intake of women living in México city. Salud Publica Mex 1998; 39(40): 133-40.

23. Potischman N, Swanson C, Coates RJ et al. Intake of food groups and associated micronutrients in relation to risk of early-stage breast cancer. Int J Cancer 1999; 82: 315-21.

24. Ocké MC, Bueno-de-Mesquita HB, Pols MA et al. The Dutch EPIC food frequency questionnaire.II. Relative validity and reproducibility for nutrients. Int J Epidemiol 1997; 26(S1): 49-58.

25. Bolschied-Thomas S, Hoting I, Boeing $\mathrm{H}$ et al. Reproducibility and relative validity of food group intake in a food frequency questionnaire developed for the German part of the EPIC Project. Int J Epidemiol 1997; 26(S1): 59-70.

26. Ling AMC, Horwath C, Parnell W. Validation of a short food frequency questionnaire to assess consumption of cereal foods, fruit and vegetables in Chinese Singaporeans. Eur J Clin Nutr 1998; 52: 557-64.

27. Schaffer DM, Coates AO, Caan BJ et al. Performance of a shortened telephone-administered version of a quantitative food frequency questionnaire. Ann Epidemiol 1997; 7(7): 463:71.
28. Gray-Donald K, O`Loughlin J, Richard L, Paradis G. Validation of a short telephone administered questionnaire to evaluate dietary interventions in low income communities in Montreal, Canadá. J Epidemiol Community Health 1997; 51(3): 326-31.

29. Lyu L, Hankin JH, Liu LQ et al. Telephone vs face-to-face interviews for quantitative food frequency asessment. $J$ Am Diet Assoc 1998; 98: 44-8.

30. Potischman N, Swanson C, Coates RJ et al. Intake of food groups and associated micronutrients in relation to risk of early-stage breast cancer. Int J Cancer 1999; 82: 315-21.

31. Subar AF, Thompson FE, Kipnis V et al. Comparative validation of Block, Willett and National Cancer Institute food frequency questionnaires. Am J Epidemiol 2001; 154(12): 1089-99.

32. Marchioni DML, Voci SM, Lima FEL de; et al. Reproducibility of a food frequency questionnaires for adolescents. Cad Saúde Pública 2007; 23(9): 2187-96.

33. Hunter DJ , Sampson 1, Stampfer MJ et al. Variability in portion sizes of commonly consumed foods among a population of women in the United States. Am J Epidemiol 1988;127(6):1240-9.

Recebido em: 22/03/07

Versão final reapresentada em: 26/10/07

Aprovado em: 09/11/07 